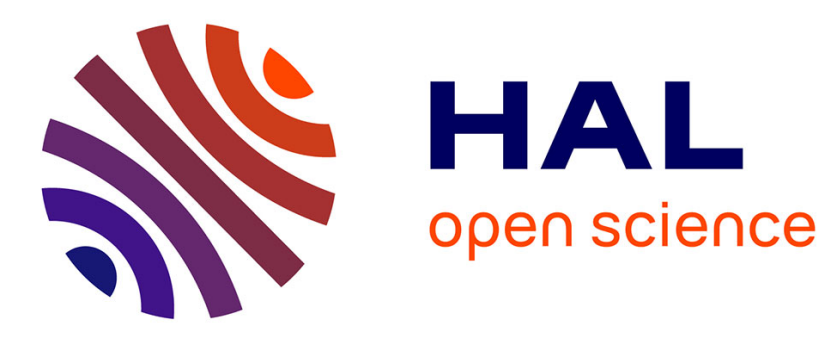

\title{
Aperiodic order and pure point diffraction
}

\author{
Daniel Lenz
}

\section{To cite this version:}

Daniel Lenz. Aperiodic order and pure point diffraction. Philosophical Magazine, 2008, 88 (13-15), pp.2059-2071. 10.1080/14786430802082008 . hal-00513889

\section{HAL Id: hal-00513889 \\ https://hal.science/hal-00513889}

Submitted on 1 Sep 2010

HAL is a multi-disciplinary open access archive for the deposit and dissemination of scientific research documents, whether they are published or not. The documents may come from teaching and research institutions in France or abroad, or from public or private research centers.
L'archive ouverte pluridisciplinaire HAL, est destinée au dépôt et à la diffusion de documents scientifiques de niveau recherche, publiés ou non, émanant des établissements d'enseignement et de recherche français ou étrangers, des laboratoires publics ou privés. 


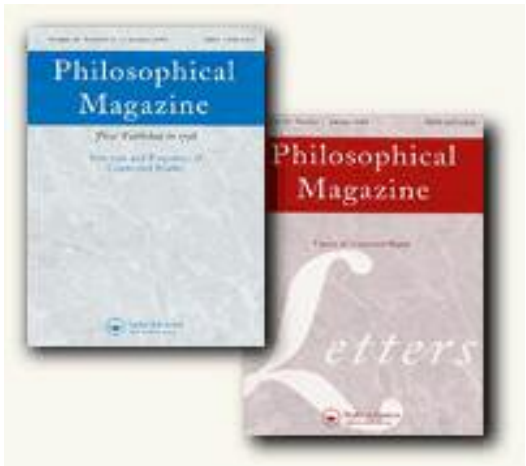

\section{Aperiodic order and pure point diffraction}

\begin{tabular}{|c|c|}
\hline Journal: & Philosophical Magazine \& Philosophical Magazine Letters \\
\hline Manuscript ID: & TPHM-07-Dec-0361.R2 \\
\hline Journal Selection: & Philosophical Magazine \\
\hline $\begin{array}{r}\text { Date Submitted by the } \\
\text { Author: }\end{array}$ & 19-Mar-2008 \\
\hline Complete List of Authors: & Lenz, Daniel; TU Chemnitz, Fakultat fur Mathematik \\
\hline Keywords: & diffraction, quasicrystals \\
\hline \multicolumn{2}{|l|}{ Keywords (user supplied): } \\
\hline \multicolumn{2}{|c|}{$\begin{array}{l}\text { Note: The following files were submitted by the author for peer review, but cannot be converted } \\
\text { to PDF. You must view these files (e.g. movies) online. }\end{array}$} \\
\hline Lenzrev3.tex & \\
\hline
\end{tabular}

\section{S) ScholaroNE \\ Manuscript Central}


Philosophical Magazine,

Vol. 00, No. 00, DD Month 200x, 1-10

\title{
Aperiodic order and pure point diffraction
}

\author{
Daniel Lenz* \\ Fakultät für Mathematik, TU Chemnitz, 09107 Chemnitz, Germany \\ Current address: Department of Mathematics, Rice University, P. O. Box 1892, Houston, TX 77251, USA \\ ()
}

\begin{abstract}
The paper gives a leisurely introduction into mathematical diffraction theory with a focus on pure point diffraction. In particular, various characterisations of pure point diffraction and common models arising from cut and project schemes are discussed. It finishes with a list of open problems.
\end{abstract}

\section{Introduction}

Quasicrystals were discovered by their unusual diffraction properties [1]. Subsequently, quite some mathematical effort has been devoted to diffraction theory of aperiodically ordered models (see corresponding parts in [2-5] and references therein). In fact, there is substantially more work done than could sensibly be reviewed here. For this reason I will focus below on pure point diffraction and model sets. These topics seem to be both particularly relevant and conceptually fairly well understood. Let me note in passing that model sets were introduced by Meyer [6] quite some time before the dawn of quasicrystals. They became a standard in the physical literature.

While recent results give a diffraction theory for measures on locally compact abelian groups [7-10], I will restrict attention here to point sets in Euclidean space in order to keep the exposition as simple as possible. The much studied topic of mixed spectra and random systems, see e.g. [11-15] and references therein, will only appear in the last section on problems.

Earlier survey type articles on mathematical diffraction are given in [16,17]. A detailed introduction into mathematical diffraction theory is given in the lecture notes [18]. A somewhat introductory text from the point of view of dynamical systems can be found in [19].

The article is organised as follows: Section 2 introduces the framework of mathematical diffraction theory and defines and discusses its key quantity, the diffraction measure. Section 3 is concerned with characterisation of pure point diffraction. Section 4 deals with cut and project schemes and model sets. Finally, the last section is devoted to some open problems.

\section{The framework of mathematical diffraction theory}

Diffraction theory for crystals has a long history. Discussions can be found in many textbooks, e.g. [20]. The first systematic treatment of mathematical diffraction theory for aperiodic order is due to Hof $[21,22]$. Here, I discuss the basic setup. The mathematical key quantity turns out to be a measure, the so called diffraction measure. It models the outcome of a diffraction experiment.

The solid in question is modeled by a subset $\Lambda$ of Euclidean space $\mathbb{R}^{N}$. It will be assumed that $\Lambda$ is distributed in a regular way with points being not too close and not too far. More precisely, $\Lambda$ is assumed to be Delone, i.e.

\footnotetext{
*Email:dlenz@mathematik.tu-chemnitz.de
} 
- there exists an $r>0$ such that different points of $\Lambda$ have distance at least $2 r$ (" $\Lambda$ is uniformly discrete"),

- there exists an $R>0$ such that no point of Euclidean space has distance bigger than $R$ from $\Lambda$ (" $\Lambda$ is relatively dense").

Thus, clearly, $\Lambda$ has infinitely many points. In order to understand the diffraction setup for the infinite $\Lambda$ it is helpful to first consider the case of a finite set $F$ of scatterers. In this case the intensity is the function given by

$$
I_{F}(\xi)=\left|\sum_{x \in F} \exp (-i x \xi)\right|^{2}=\sum_{x, y \in F} \exp (-i(x-y) \xi)
$$

The analogous expression for the infinite $\Lambda$ reads

$$
\sum_{x, y \in \Lambda} \exp (-i(x-y) \xi)
$$

This sum is heavily divergent, as there are infinitely many terms of modulus 1 . This is not a mathematical problem only. There is a physical reason behind this divergence: The intensity of the infinite solid is infinite. The correct object to be considered is the normalised intensity per unit volume. It is defined as

$$
I_{\Lambda}=\lim _{n \rightarrow \infty} \frac{1}{\left|B_{n}\right|} I_{\Lambda \cap B_{n}}
$$

Here, $B_{n}$ denotes the ball around the origin with radius $n$ and the modulus denotes the volume. Note that $\Lambda \cap B_{n}$ is finite by our assumption on $\Lambda$ and, hence, $I_{\Lambda \cap B_{n}}$ is a well defined function. There are issues in (2.1) in which sense the limit is meant (it is the vague sense) and whether it exists at all (one may have to pass to a subsequence). However, I will skip these somewhat technical details.

The quantity $I_{\Lambda}$ is known as diffraction measure. It describes the outcome of a diffraction experiment. The values $\xi$ with $I_{\Lambda}(\{\xi\})>0$ are called Bragg peaks. The value of $I_{\Lambda}(\{\xi\})$ is called intensity of the Bragg peak.

Now, that $I_{\Lambda}$ is defined one may wonder how to calculate it. To this end let me expand the definition of $I_{\Lambda}$. This gives

$$
\begin{aligned}
I_{\Lambda}=\lim _{n \rightarrow \infty} \frac{1}{\left|B_{n}\right|} I_{\Lambda \cap B_{n}} & =\lim _{n \rightarrow \infty} \frac{1}{\left|B_{n}\right|} \sum_{x, y \in \Lambda \cap B_{n}} \exp (-i(x-y) \xi) \\
& =\lim _{n \rightarrow \infty} F\left(\frac{1}{\left|B_{n}\right|} \sum_{x, y \in \Lambda \cap B_{n}} \delta_{x-y}\right) \\
& =F\left(\lim _{n \rightarrow \infty} \frac{1}{\left|B_{n}\right|} \sum_{x, y \in \Lambda \cap B_{n}} \delta_{x-y}\right) .
\end{aligned}
$$

Here, $\delta_{x}$ denotes the unit point mass at $x$ and $F$ is the Fourier transform. This calculation shows that $I_{\Lambda}$ is the Fourier transform of

$$
\gamma_{\Lambda}=\lim _{n \rightarrow \infty} \frac{1}{\left|B_{n}\right|} \sum_{x, y \in \Lambda \cap B_{n}} \delta_{x-y}
$$

This object $\gamma_{\Lambda}$ is known as autocorrelation or Patterson function (though it is a measure).

In a theoretical sense the investigation of diffraction is thus reduced to the following two step procedure: (1) Calculate the Patterson function. (2) Take its Fourier transform. 
While it is possible to successfully carry out this procedure for various examples, it is far from obvious how to do this in the general case. For this reason a lot of effort has been put into finding general criteria for the investigation of point spectra. The basic questions to be addressed are the following:

- When is $I_{\Lambda}$ a pure point measure?

- Where are the Bragg peaks?

- What are the intensities of the Bragg peaks?

Answers to these questions will be provided in subsequent sections.

\section{Pure point diffraction}

On the conceptual level two approaches to pure point diffraction have been developed in the last ten or so years. These are given, respectively, by

- considering the associated dynamical systems, or

- investigating almost periodicity properties.

In this section I discuss these two approaches.

\subsection{Pure point diffraction via dynamical systems}

When dealing with disordered systems in statistical mechanics it is quite standard not to consider a single object but a whole ensemble of objects exhibiting the "same type of disorder". In the realm of aperiodic order the same reasoning can be applied. It suggests to consider not a single set $\Lambda$ but rather the ensemble of all subsets $\Gamma$ of Euclidean space which have the same local structure as $\Lambda$. This ensemble will be denoted by $\Omega$. It can be made into a compact topological space [23-25] but I will not worry about this here. Instead I will note the following crucial feature of $\Omega$ : If $\Gamma$ belongs to $\Omega$ then so does its translate by $t \in \mathbb{R}^{N}$ given as

$$
t+\Gamma=\{t+x: x \in \Gamma\}
$$

The obvious reason is that $\Gamma$ and $t+\Gamma$ have the same local structure. Thus, $\Omega$ together with the translations gives a dynamical system. I will write $\left(\Omega, \mathbb{R}^{N}\right)$ to denote this structure.

In many situations $\Omega$ comes with a canonical translation invariant measure $m$. In these cases it is possible to provide an autocorrelation $\gamma=\gamma_{m}$ by a closed formula invoking only $m$ and not using a limit. This was first realized by Gouéré [26]. While his further considerations use the theory of stochastic processes and Palm measures, the formula for $\gamma_{m}$ can rather directly be given. Following Baake/Lenz [7] one obtains for the application of the measure $\gamma_{m}$ to the continuous compactly supported function $\varphi$

$$
\gamma_{m}(\varphi)=\int_{\Omega} \sum_{x, y \in \Gamma} \varphi(x) \sigma(x-y) d m(\Gamma),
$$

where $\sigma$ is a function on $\mathbb{R}^{N}$ with $\int \sigma(t) d t=1$. In this way the autocorrelation is defined without a limit. It can then be shown that it actually agrees with the corresponding limit almost surely $[7,26]$.

In various prominent cases $\Omega$ is actually uniquely ergodic, i.e. there exists a unique translation invariant probability measure on $\Omega$. Then, the autocorrelation exists for all elements in $\Omega$. If there are only finitely many patterns of each size then unique ergodicity can be characterised combinatorially by uniform existence of the pattern frequencies, see e.g. [24,25].

Whenever $\Omega$ is equipped with an invariant measure $m$ it is further possible to consider the associated space $L^{2}(\Omega, m)$ of square integrable functions on $\Omega$. The translations on $\Omega$ induce a unitary representation 
of $\mathbb{R}^{N}$ on $L^{2}(\Omega, m)$ via

$$
\left(T_{t} f\right)(\Gamma)=f(t+\Gamma)
$$

for $t \in \mathbb{R}^{N}$. A function $f \in L^{2}(\Omega, m)$ is called an eigenfunction to the eigenvalue $\xi$ if

$$
T_{t} f=\exp (i t \xi) f
$$

for all $t \in \mathbb{R}^{N}$. If there exists a basis of $L^{2}(\Omega, m)$ consisting of eigenfunctions, the dynamical system $\left(\Omega, \mathbb{R}^{N}\right)$ is said to have pure point spectrum. The main result on pure point diffraction in the context of dynamical systems now reads as follows.

TheOREM 3.1 Let $\left(\Omega, \mathbb{R}^{N}\right)$ together with an invariant measure $m$ be given. Then, the following assertions are equivalent:

(i) The Fourier transform $I_{m}$ of $\gamma_{m}$ is a pure point measure.

(ii) The dynamical system $\left(\Omega, \mathbb{R}^{N}\right)$ with the measure $m$ has pure point spectrum.

In this case the group generated by the Bragg peaks $\left\langle\left\{\xi: I_{m}(\{\xi\})>0\right\}\right\rangle$ is the group of eigenvalues of the dynamical system.

This theorem gives a characterisation of pure point diffraction in terms of the dynamical system. Moreover, it provides further information on the position of the Bragg peaks. The implication $(i i) \Longrightarrow(i)$ of the theorem established in [27] has been a basic tool in establishing pure point diffraction for concrete classes of models $[21,24,28,29]$. The converse implication $(i) \Longrightarrow(i i)$ allows one to set up a perturbation theory for pure point diffraction in the context of dynamical systems. This has been carried out in [8] (see [30] for related material as well). A particular application is the study of deformed model sets, as done earlier in $[31,32]$.

The theorem is the outcome of cumulated efforts over many years: The analogous result for one dimensional subshifts was proven by Quefféléc in [33]. For the dynamical systems considered here, the implication $(i i) \Longrightarrow(i)$ is due to Dworkin [27] with later modifications by Schlottmann [28] and Hof [22] (see [34] for related material as well). The full equivalence was proven for systems satisfying a certain regularity assumption known as finite local complexity by Lee/Moody/Solomyak in [35]. For the systems considered here (and even more general ones) the full equivalence was then shown by Gouéré [32]. It is possible to leave the class of point processes and to go to measures instead as discussed in Baake/Lenz [7] and then also in Lenz/Strungaru [36]. The statement on the eigenvalues is implicit in the proof of [35]. An explicit formulation can be found in [7].

The theorem rises the question whether the spectrum of the dynamical system is always given by the diffraction spectrum. This turns out to be wrong as was shown by van Enter/Miękisz [34]. More precisely, the dynamical spectrum always contains the diffraction spectrum [27]. However, it maybe strictly richer than the diffraction spectrum as shown by examples [34].

The theorem does not answer the question on the intensities of the Bragg peaks. The basic physical intuition concerning this issue is that the intensity of the Bragg peak at $\xi$ should be given by

$$
I_{\Lambda}(\{\xi\})=\lim _{n \rightarrow \infty}\left|\frac{1}{\left|B_{n}\right|} \sum_{x \in \Lambda \cap B_{n}} \exp (-i x \xi)\right|^{2} .
$$

Validity of this equation is sometimes discussed under the header of Bombieri/Taylor conjecture. In fact Bombieri/Taylor use validity of this equation in their work $[37,38]$ without any further justification. By now validity has been established by direct arguments for primitive substitution systems by Gähler/Klitzing [39] and for regular models sets $[21,28]$. In fact, these results follow from a conceptual approach to (3.1) via continuity of eigenfunctions and uniform Wiener/Wintner type results. This has been developed by Lenz [40]. The conceptual approach itself is hinted at in $[16,22]$. The necessary continuity of eigenfunctions 
is proven for model sets in [22,28] and for primitive substitutions by Solomyak in [41]. The corresponding Wiener/Wintner type results had already been studied by Robinson [42].

\subsection{Pure point diffraction and almost periodicity}

In some way or other notions of almost periodicity have been around in the study of pure point diffraction for quite a while. In particular, there is work of Solomyak [43] providing a connection and a discussion of Lagarias [16] asking for connections. There is even a characterisation of pure point diffraction in terms of almost periodicity in the work of Quefféléc in [33] for the (somewhat different) situation of symbolic dynamics. Furthermore, Meyer's work on what is now known as Meyer sets [6] and subsequent discussions, see e.g. [44], have a rather almost periodic flavour. Still it seems fair to say that only with the rather recent work of Baake/Moody [9] and Gouéré [32] the strength of this connection became apparent.

A continuous function $f$ on $\mathbb{R}^{N}$ is called almost-periodic if for any $\varepsilon>0$ the set of its $\varepsilon$-almost-periods

$$
\left\{t \in \mathbb{R}^{N}:\|f(\cdot-t)-f\|_{\infty} \leq \varepsilon\right\}
$$

is relatively dense in $\mathbb{R}^{N}$. Here, $\|\cdot\|_{\infty}$ denotes the supremum norm. Similar notions for measures exist and give the concept of strongly-almost-periodic measure and norm-almost-periodic measure. A measure is called strongly-almost-periodic if its convolutions with continuous compactly supported functions are almost-periodic. Norm-almost-periodicity is defined using the total variation of the measure. While I do not want to further concern the reader with the technical definitions here, I would like to note that norm almost periodicity is substantially stronger than strong almost periodicity.

It is also possible to develop a concept of almost periodicity for sets. This is done under the name of Bohr/Besicovitch almost periodicity by Gouéré [32] (see corresponding questions of Lagarias in [16] as well).

TheOrem 3.2 Let $\Lambda$ be a Delone set whose autocorrelation $\gamma_{\Lambda}$ exists. Then, the following assertions are equivalent:

(i) The Fourier transform $I_{\Lambda}$ of $\gamma_{\Lambda}$ is a pure point measure.

(ii) $\gamma_{\Lambda}$ is a strongly-almost-periodic measure.

(iii) $\Lambda$ is Bohr/Besicovich almost periodic.

For sets $\Lambda$ whose autocorrelation is supported on a uniformly discrete set, the result is proven by Baake/Moody [9]. In the general form stated above this theorem is due to Gouéré [32].

As will be discussed in the next section, one is particularly interested in the case of Meyer sets $\Lambda$. These are Delone sets with the property that $\Lambda-\Lambda$ is uniformly discrete. For such sets it makes sense to define an $\varepsilon$-almost period or better a statistical $\varepsilon$-almost period as a $t \in \mathbb{R}^{N}$ with

$$
\limsup _{n \rightarrow \infty} \frac{\sharp(\Lambda \backslash(\Lambda+t) \cup(\Lambda+t) \backslash \Lambda)) \cap B_{n}}{\left|B_{n}\right|} \leq \varepsilon .
$$

Here, $\sharp$ denotes cardinality. For Meyer sets Baake/Moody [9] have the following result.

THEOREM 3.3 Let $\Lambda$ be Meyer with autocorrelation $\gamma_{\Lambda}$. Then, the following assertions are equivalent:

(i) The Fourier transform $I_{\Lambda}$ of $\gamma_{\Lambda}$ is a pure point measure.

(ii) $\gamma_{\Lambda}$ is a norm-almost-periodic measure.

(iii) For any $\varepsilon>0$ the set of statistical almost- $\epsilon$-periods of $\Lambda$ is relatively dense.

Let me emphasise that the actual setting and results of [9] are considerably more general than discussed in this theorem. In particular, [9] deals with weighted point sets on locally compact abelian groups. Moreover, it gives a natural connection between pure point diffraction and cut and project scheme (see below for details). 


\section{Cut and project schemes and model sets}

The two most prominent classes of mathematical models for aperiodic order are models coming from primitive substitutions and models coming from cut and project schemes. The latter are often discussed under the name of model sets (see e.g. [44,45] for further discussion and references). They provide also standard examples discussed in the physical literature. There is a wealth of results on model sets and cut and project schemes. Here, I focus on the following issues:

- explicit computation of $I_{\Lambda}$ for regular model sets, carried out by Hof [21,22] (see later generalisations $[9,28]$ as well),

- existence of a lot of point diffraction for general sets associated to cut and project schemes, shown by Strungaru [46],

- a natural connection between cut and project schemes and pure point diffraction, discovered by Baake/Moody [9], and then further explored in [47-49],

- characterisation of primitive substitutional sets with pure point diffraction as model sets, due to Lee [50] (see the work of Barge/Kwapisz [51,52] for an analogous one dimensional result in a slightly different context).

The first result justifies mathematically the corresponding parts of the physical literature. The second result shows that order in the sense of Meyer condition implies order in the sense of a large point component in the diffraction spectrum. The third result (or rather the corresponding circle of ideas) shows that cut and project sets arise very naturally within the framework of pure point diffraction for Meyer type sets. The final result shows that the "other class of examples" viz primitive substitutions is not really a different class when it comes to models with pure point diffraction.

Let me now start by shortly recalling the framework of a cut and project scheme. Besides the physical space $\mathbb{R}^{N}$ a cut and project scheme has two further ingredients. These are a further space and a lattice. The further space is known as internal space, perpendicular space or reciprocal space. It will be denoted by $H$. It does not need to be an Euclidean space. It suffices if it is a locally compact Abelian group. The lattice is denoted by $\widetilde{L}$. It is a lattice in $\mathbb{R}^{N} \times H$. Its projections to the physical space and the internal space will be denoted by $L$ and $L^{\star}$ respectively. A precise definition of cut and project scheme now runs as follows.

A cut and project scheme over $\mathbb{R}^{N}$ consists of a locally compact Abelian group $H$, and a lattice $\widetilde{L}$ in $\mathbb{R}^{N} \times H$ such that the canonical projection $\pi: \mathbb{R}^{N} \times H \longrightarrow \mathbb{R}^{N}$ is one-to-one between $\tilde{L}$ and $L:=\pi(\widetilde{L})$ and the image $\pi_{\text {int }}(\widetilde{L})$ of the canonical projection $\pi_{\text {int }}: \mathbb{R}^{N} \times H \longrightarrow H$ is dense. Given these properties of the projections $\pi$ and $\pi_{\text {int }}$, one can define the $\star$-map $(.)^{\star}: L \longrightarrow H$ via $x^{\star}:=\left(\pi_{\text {int }} \circ\left(\left.\pi\right|_{L}\right)^{-1}\right)(x)$, where $\left(\left.\pi\right|_{L}\right)^{-1}(x)=\pi^{-1}(x) \cap \tilde{L}$, for all $x \in L$. One can summarise the features of a cut and project scheme in the following diagram:

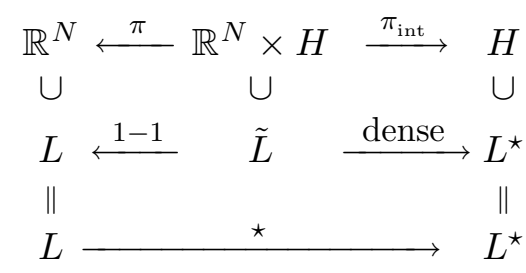

We will assume that the Haar measures on $\mathbb{R}^{N}$ and on $H$ are chosen in such a way that a fundamental domain of $\tilde{L}$ has measure 1 . Given a cut and project scheme, one can associate to any $W \subset H$, called the window, atomic surface or acceptance domain, the set

$$
\text { 人 }(W):=\left\{x \in L: x^{\star} \in W\right\}
$$

A set of the form $t+\boldsymbol{\curlywedge}(W)$ is called model set if the window $W$ is relatively compact with nonempty interior. 
Theorem 4.1 Let $\Lambda$ be a Delone set. Then, the following assertions are equivalent:

(i) $\Lambda-\Lambda$ is uniformly discrete.

(ii) $\Lambda$ is a subset of a model set.

(iii) There exists a finite set $F$ with $\Lambda-\Lambda \subset \Lambda+F$.

The equivalence of (i) and (ii) is due to Meyer [6] and Moody [44]. The equivalence of (i) and (iii) is due to Lagarias [53]. The sets characterised in the previous theorem are known as Meyer sets. Note that all three conditions appearing in the theorem can be understood as indicating long range order in form of a weak lattice type condition. Various further characterisations can be found in the literature $[44,53]$.

Let me mention that a model set is relatively dense due to non-emptyness of the interior of the window and uniformly discrete by compactness of the window. This compactness then also gives condition (i) of the previous theorem for model sets. Thus, each model set is Meyer. The converse is not true (as can easily be seen by removing points from a model set). However, part (ii) of the previous theorem shows that each Meyer set arises from a model set after (possibly) deleting points.

\subsection{An explicit formula for $I_{\Lambda}$}

Let a cut and project scheme $\left(\mathbb{R}^{N}, H, \widetilde{L}\right)$ be given. Let also a sufficiently nice window $W$ in $H$ be given. Sufficiently nice means roughly speaking that the window is not a fractal. More precisely, $W$ is required to be compact with non empty interior and and a boundary of measure zero. In this case, one can calculate explicitely the diffraction measure $I_{\Lambda}[21,28]$. The dual lattice $\widetilde{L}^{\perp}$ of $\widetilde{L}$ is given by

$$
\widetilde{L}^{\perp}:=\left\{(k, u) \in \widehat{\mathbb{R}^{N}} \times \widehat{H}: e^{i k l} u\left(l^{\star}\right)=1 \text { for all }\left(l, l^{\star}\right) \in \widetilde{L}\right\}
$$

Let $L^{\circ}$ be the set of all $k \in \mathbb{R}^{N}$ for which there exists $u \in \widehat{H}$ with $(k, u) \in \widetilde{L}^{\perp}$. This set is sometimes known as reciprocal lattice. It can be shown that there exists a unique group homomorphism $\star: L^{\circ} \longrightarrow \widehat{H}$ such that

$$
\tau: L^{\circ} \longrightarrow \widetilde{L}^{\perp}, \quad k \mapsto\left(k, k^{\star}\right)
$$

is bijective. Then, the diffraction measure $I_{\Lambda}$ is given by

$$
\widehat{\gamma_{\Lambda}}=\sum_{k \in L^{\circ}} A_{k} \delta_{k}, \text { with } A_{k}=\left|\int_{W}\left(k^{\star}, y\right) d y\right|^{2}
$$

If $H$ happens to be an Euclidean space as well, the formula for $A_{k}$ reads $\left|\int_{W} \exp \left(i k^{\star} y\right) d y\right|^{2}$. Note that the formulas for the $A_{k}$ are particularly simple as the lattice $\widetilde{L}$ is normalised to have fundamental domain with volume 1.

\subsection{A lot of point diffraction for Meyer sets}

This subsection highlights the following result of Strungaru [46].

TheOREM 4.2 Let $\Lambda$ be Meyer with autocorrelation $\gamma_{\Lambda}$. Then, $\widehat{\gamma_{\Lambda}}$ has a relative dense set of Bragg peaks.

As mentioned already this result can be understood as linking two notions of long range order. The result is rather general as it does not assume any further regularity properties of the point set in question.

\subsection{A natural cut and project scheme}

It is a fundamental insight of Baake/Moody [9] that any set with a sufficiently almost periodic autocorrelation comes with a natural cut and project scheme. The required almost periodicity of the autocorrelation 
in turn is equivalent to pure point diffraction whenever the autocorrelation is supported on a uniformly discrete set. This ties pure point diffraction and cut and project schemes (within the context of Meyer type sets).

A crucial step in the argument of [9] is to use the autocorrelation function to introduce a topology on point sets. Taking completions with respect to this topology then yields the internal space. In this way, cut and project schemes lie at the crossroads of two topologies: the local topology and the topology coming from the autocorrelation function. This point of view is further developed in $[47,49,54]$.

These results allow one to derive the characterisation of pure point diffractiveness for Meyer sets given in Theorem 3.3 above. They can also be used to characterise the dynamical systems arising from regular model sets. This has been discussed by Baake/Lenz/Moody [48]. As [9] provides a cut and project scheme, the main task in [48] is to construct and study the window using properties of the dynamical system. The basic connection between the dynamical system and the cut and project scheme is given by the so called torus parametrisation $[28,55]$. The torus parametrisation turns out to be strongly determined by properties of the eigenfunctions. Two properties of the eigenfunctions are central. These are continuity of eigenfunctions and their separation properties.

As a byproduct one obtains a characterisation of lattices within Meyer sets with pure point diffraction. The work [48] also plays an important role in the investigation of substitution systems discussed next.

\subsection{Substitutions with pure point diffraction}

As mentioned already the most important classes of examples for aperiodic order are model sets and sets arising from primitive substitution. Here, I will shortly discuss a remarkable result of Lee [50] (see [51] as well) relating primitive substitution sets to model sets within the context of pure point diffraction. In some sense the result shows that one can not get away from model sets when dealing with pure point diffraction. More precisely, Theorem 5.5 of [50] gives in particular the following.

THEOREM 4.3 Let $\Lambda$ be induced by a primitive substitution with finite local complexity. Then, the following assertions are equivalent:

(i) $\Lambda$ has pure point dynamical spectrum.

(ii) $\Lambda$ is an inter model set.

The notion of a set associated to a primitive substitution requires some care. One possibility is to consider tilings arising as fixed points of a primitive substitution. Then a point is chosen in each tile so that points for tiles of the same type are in the same relative position in the tile. Thinking of the points in different classes of tiles as marked with different colours one obtains a coloured set. Such a set is underlying the statement of the previous theorem. The condition (i) is equivalent to a (suitably defined) notion of pure point diffraction for coloured sets. The condition (ii) also has to be understood for coloured sets. By definition an inter model set agrees with a model set up to points induced by the boundary of the window. Instead of using tilings one can directly deal with substitutions for point sets, see Theorem 5.3 in [50]. In this case the additional assumption of legality of certain clusters has to be imposed.

The proof of these results winds together different strings of reasoning. One such string concerns so called coincidence conditions. They give criteria for a primitive substitution to have pure point diffraction. Building up on earlier work $[56,57]$ Lee gives a new coincidence condition allowing for a characterisation of pure point diffraction for primitive substitutions. This characterisation is in fact part of the main result. It is done under the assumption that the set in question is Meyer. The second string then is a result of Lee/Solomyak [58] showing that a primitive substitution with pure point diffraction must be Meyer. As a third ingredient Lee uses the recent results of Baake/Lenz/Moody [48] providing a characterisation of regular model sets in terms of the associated dynamical systems.

\section{Open questions}

In this section I present various issues and questions for further research. 
- Geometric implications of pure point diffraction.

The phenomenon of pure point diffraction seems to be fairly well understood within the context of Meyer sets. This poses the question whether pure point diffraction in itself together with mild geometric restrictions (as e.g. finite local complexity and repetitivity) forces the Meyer property. For primitive substitutions this has been answered affirmatively by Lee/Solomyak [58]. The general case seems to be open. A particular instance of this type of issue is the question which properties single out the lattices within the sets with pure point diffraction. For further discussion of these and related issues I refer to the article of Lagarias [16].

A further issue in this context is the validity of Bombieri/Taylor conjecture (discussed above) for larger classes of examples.

Another question concerns entropy. Of course, entropy should somehow vanish for models with aperiodic order. Indeed, sets with pure point diffraction and further regularity can be shown to have vanishing topological entropy [59]. On the other hand, there are natural examples of sets with pure point diffraction exhibiting positive topological entropy. Such an example is given by the set of visible points as shown by Pleasants [60]. It seems that this is related to cut and project schemes with a window with a "thick" boundary.

- Mixed spectra and random systems.

The understanding of mixed spectra is very much at the beginning. Let me illustrate this by considering two extreme cases: On the one hand there are primitive substitutions models. These models carry a lot of order by their very construction. Still, not all primitive substitutions have point spectrum, let alone pure point spectrum. Thus, mixed spectra go well with a very rigid order structure. This is a conceptual issue in the understanding of order as encoded by spectral properties. On the other hand there are random systems. Random systems based on lattices exhibit a tendency to have mixed spectra with a pure point component due to the lattice and an absolutely continuous component due to the randomness. While this is well confirmed in examples $[12,13]$ a general treatment is not available yet.

Actually, random systems and substitutions are not that far apart in terms of diffraction. More precisely, as discussed by Baake/Hoeffe [12] it is possible to have a primitive substitution system with the same diffraction as a random system.

One reason that diffuse spectra are not as well understood as point spectra is that there does not seem to be a good dynamical interpretation.

- Homometry and inverse problem.

The above considerations have been concerned with the direct problem i.e. to determine the diffraction given the solid. Of course, the real problem is the inverse problem. In mathematical terms this amounts to describing all configurations leading to the same diffraction. This is known as the homometry problem. In this context one may ask for properties shared by all solutions to the inverse problem as well as for further restrictions making the solution unique.

Acknowledgements. It is my pleasure to acknowledge helpful discussions with Michael Baake when I was preparing the talk at "Quasicrystals - The Silver Jubilee", Tel Aviv, 2007, out of which this article arose. I take this opportunity to thank the organisers for the invitation to a highly enjoyable conference. Financial support from German Science Foundation (DFG) is gratefully acknowledged.

\section{References}

[1] D. Shechtman, I. Blech, D. Gratias and J.W. Cahn, Metallic phase with long-range orientational order and no translation symmetry, Phys. Rev. Lett. 53 (1984) 183-185.

[2] M. Baake, R.V. Moody (eds.), Directions in Mathematical Quasicrystals, CRM Monograph Series, 13, AMS, Rhode Island (2000).

[3] R.V. Moody (ed.), The Mathematics of Long-Range Aperiodic Order, NATO ASI Series C 489, Kluwer, Dordrecht (1997).

[4] M. Senechal, Quasicrystals and geometry, Cambridge University Press, Cambridge, (1995).

[5] H.-R. Trebin (ed.), Quasicrystals - Structure and Physical Properties, Wiley-VCH, Weinheim (2003).

[6] Y. Meyer, Algebraic numbers and harmonic analysis. North-Holland Mathematical Library, Vol. 2. North-Holland Publishing Co., Amsterdam-London; American Elsevier Publishing Co., Inc., New York, 1972. 
[7] M. Baake, D. Lenz, Dynamical systems on translation bounded measures: Pure point dynamical and diffraction spectra, Ergodic Th. \& Dynam. Syst. 24 (2004), 1867-1893.

[8] M. Baake, D. Lenz, Deformation of Delone dynamical systems and pure point diffraction, J. Fourier Anal. Appl. 11 (2005), 125-150.

[9] M. Baake, R.V. Moody, Weighted Dirac combs with pure point diffraction, J. Reine Angew. Math. (Crelle) 573 (2004), 61-94; math.MG/0203030.

[10] D. Lenz, C. Richard, Cut and project schemes for measures: the smooth case, Mathematische Zeitschrift 256, (2007), 347-378.

[11] M. Baake, D. Frettloeh, U. Grimm, Pinwheel patterns and powder diffraction, Philosophical Magazine 87 (2007), $2831-2838$.

[12] M. Hoeffe, M. Baake Surprises in diffuse scattering, Z. Kristallogr. 215 (2000), 441-444.

[13] M. Baake, B. Sing, Diffraction spectrum of lattice gas models above $T_{c}$, Lett. Math. Phys. 68 (2004), $165-173$.

[14] R.V. Moody, D. Postnikoff, N. Strungaru, Circular symmetry of pinwheel diffraction, Ann. Henri Poincaré 7 (2006), 711-730.

[15] C. Radin, Symmetries of quasicrystals, J. Stat. Phys. 95 (1999), 827-833.

[16] J. C. Lagarias, Mathematical quasicrystals and the problem of diffraction, in [2], 61-93.

[17] M. Baake, R. V. Moody, C. Richard, B. Sing: Which distributions of matter diffract? Some answers, in: [5] $188-207$.

[18] M. Baake, Mathematical Diffraction in Euclidean Spaces, Lecture Notes EPFL Lausanne, available at: http://cristallo.epfl.ch/3cycle/courses/Baake-2004.pdf

[19] D. Lenz, Aperiodic order via dynamical systems: Diffraction for sets of finite local complexity, preprint, arXiv:0712.1323.

[20] J.M. Cowley, Diffraction Physics, 3rd ed., North-Holland, Amsterdam (1995).

[21] A. Hof, On diffraction by aperiodic structures, Commun. Math. Phys. 169 (1995), 25-43.

[22] A. Hof, Diffraction by aperiodic structures, in: [3], pp. 239-268.

[23] C. Radin, M. Wolff, Space tilings and local isomorphism, Geom. Dedicata 42 (1992), 355-360.

[24] B. Solomyak, Dynamics of self-similar tilings, Ergodic Th. \& Dynam. Syst. 17 (1997), 695-738; Erratum: Ergodic Th. \& Dynam. Syst. 19 (1999), 1685.

[25] J. C. Lagarias, P. A. B. Pleasants, Repetitive Delone sets and quasicrystals, Ergodic Theory Dynam. Systems 23 (2003), no. 3 , $831-867$.

[26] J.-B. Gouéré, Diffraction and Palm measure of point processes, C. R. Acad. Sci. Paris (2004),

[27] S. Dworkin, Spectral theory and X-ray diffraction, J. Math. Phys. 34 (1993), 2965-2967.

[28] M. Schlottmann, Generalized model sets and dynamical systems, in: [2], pp. 143-159.

[29] E.A. Robinson, The dynamical properties of Penrose tilings, Trans. Amer. Math. Soc., 348, 4447-4464.

[30] A. Clark, L. Sadun, When shape matters: deformations of tiling spaces, Ergodic Theory \& Dynam. Systems 26 (2006), 69-86.

[31] G. Bernuau, M. Duneau, Fourier analysis of deformed model sets, in [2], 43-60.

[32] J.-B. Gouéré, Quasicrystals and almost periodicity, Commun. Math. Phys. 255 (2005), 655-681.

[33] M. Quéffélec, Substitution dynamical systems - spectral analysis, Lecture Notes in Mathematics, 1294. Springer-Verlag, Berlin, 1987.

[34] A. C. D. van Enter, J. Miękisz, How should one define a (weak) crystal?, J. Stat. Phys. 66 (1992), 1147-1153.

[35] J.-Y. Lee, R.V. Moody, B. Solomyak, Pure point dynamical and diffraction spectra, Annales Henri Poincaré 3 (2002), 1003-1018; mp_arc/02-39.

[36] D. Lenz, N. Strungaru, Pure point spectrum for measure dyamical systems on locally compact Abelian groups, preprint, arXiv:0704.2498.

[37] E. Bombieri, J. E. Taylor, Which distributions of matter diffract? An initial investigation, International workshop on aperiodic crystals (Les Houches, 1986). J. Physique 47 (1986), no. 7, Suppl. Colloq. C3, C3-19-C3-28.

[38] E. Bombieri, J. E. Taylor, Quasicrystals, Tilings and Algebraic numbers, in: Contemporary Mathematics 64, Amer. Math. Soc. Providence, RI, (1987), 241-264.

[39] F. Gähler, R. Klitzing, The diffraction pattern of self-similar tilings in [3], 141-174.

[40] D. Lenz, Continuity of eigenfunctions of uniquely ergodic dynamical systems and intensity of Bragg peaks, preprint, arXiv:math$\mathrm{ph} / 0608026$

[41] B. Solomyak, Eigenfunctions for substitution tiling systems, preprint, 2005.

[42] E. A. Robinson, On uniform convergence in the Wiener-Wintner theorem, J. London Math. Soc. 49 (1994), $493-501$.

[43] B. Solomyak, Spectrum of dynamical systems arising from Delone sets, in: [?] pp. 265-275.

[44] R.V. Moody, Meyer sets and their duals, in: [3], pp. 403-441.

[45] R.V. Moody, Model sets: A Survey, in: From Quasicrystals to More Complex Systems, eds. F. Axel, F. Dénoyer and J. P. Gazeau, EDP Sciences, Les Ulis, and Springer, Berlin (2000), pp. 145-166.

[46] N. Strungaru, Almost periodic measures and long-range order in Meyer sets, Discrete Comput. Geom. 33 (2005), 483-505.

[47] R.V. Moody, N. Strungaru, Point sets and dynamical systems in the autocorrelation topology, Canad. Math. Bull. 47 (2004), 82-99.

[48] M. Baake, D. Lenz, R.V. Moody, A characterisation of model sets by dynamical systems, Ergodic Theory \& Dynamical Systems 27, (2007), 341-382

[49] J.-Y. Lee, R. V. Moody, A characterization of model multi-colour sets, Ann. Henri Poincaré 7 (2006), 125-143.

[50] J.-Y. Lee, Substitution Delone sets with pure point spectrum are inter-model sets, Journal of Geometry and Physics, $\mathbf{5 7}$ (2007), $2263-2285$

[51] M. Barge, J. Kwapisz, Geometric theory of unimodular Pisot substitutions, Amer. J. Math. 128 (2006), $1219-1282$.

[52] J. Kwapisz, Geometric coincidence conjecture and pure dicrete spectrum for unimodular tiling spaces, talk given at the meeting Aperiodic Order: Dynamical Systems, Combinatorics, and Operators, Banff, Canada, 2004.

[53] J. C. Lagarias, Geometric models for quasicrystals I. Delone sets of finite type, Discrete Comput. Geom. 21 (1999), 161-191.

[54] R.V. Moody, Mathematical quasicrystals: a tale of two topologies, XIVth International Congress on Mathematical Physics, 68-77, World Sci. Publ., Hackensack, NJ, 2005.

[55] M. Baake, J. Hermisson, P. A. B. Pleasants, The torus parametrization of quasiperiodic LI-classes, J. Phys. A 30 (1997), 3029-3056

[56] J.-Y. Lee, R. V. Moody, Lattice substitution systems and model sets, Discrete Comput. Geom. 25 (2001), $173-201$.

[57] J.-Y. Lee, R.V. Moody, B. Solomyak, Consequences of pure point diffraction spectra for multiset substitution systems, Discrete Comput. Geom. 29 (2003), 525-560.

[58] J.-Y. Lee, B. Solomyak, Pure point diffractive substitution Delone sets have the Meyer property, to appear in: Disc. Comput. Geom.

[59] M. Baake, D. Lenz, C. Richard, Pure point diffraction implies zero entropy for Delone sets with uniform cluster frequencies, Lett. Math. Phys. 82 (2007), 61-77.

[60] P. A. B. Pleasants, Entropy of visible points and kth-power-free numbers, preprint (2006). 